\title{
A randomised controlled trial of a mindfulness intervention for men with advanced prostate cancer
}

Suzanne K Chambers ${ }^{1,2,3,4^{*}}$, David P Smith ${ }^{4,5}$, Martin Berry ${ }^{4,6}$, Stephen J Lepore ${ }^{7}$, Elizabeth Foley ${ }^{8}$,
Samantha Clutton ${ }^{2,4}$, Robert McDowall ${ }^{2,4}$, Stefano Occhipinti ${ }^{4,9}$, Mark Frydenberg ${ }^{4,10,11}$ and Robert A Gardiner ${ }^{4,12,13}$

\begin{abstract}
Background: Prostate cancer is the most common male cancer in developed countries, and in Australia approximately one-fifth of men with prostate cancer have advanced disease. By comparison to men with localised prostate cancer, men with advanced disease report higher levels of psychological distress; poorer quality of life; and have an increased risk of suicide. To date no psychological intervention research specifically targeting men with advanced prostate cancer has been reported. In this paper we present the protocol of a current randomised controlled trial to assess the effectiveness of a professionally-led mindfulness-based cognitive therapy (MBCT) group intervention to improve psychological well-being in men with advanced prostate cancer.

Methods/design: Ninety-five men per condition (190 men in total) will be recruited through clinicians in the Australian and New Zealand Urogenital and Prostate Cancer Trials Group and in major treatment centres in Queensland, New South Wales, Victoria and Western Australia. Patients are randomised to: (1) tele-based MBCT intervention or (2) patient education. A series of previously validated and reliable self-report measures will be administered to men at four time points: baseline/recruitment, and at 3, 6, and 9 months after recruitment and intervention commencement. Engagement with the principles of mindfulness and adherence to practice will be included as potential mediators of intervention effect. Primary outcomes are anxiety, depression and cancer-specific distress. Secondary outcomes are health-related quality of life (QoL) and benefit finding. Disease variables (e.g. cancer grade, stage) will be assessed through medical records.
\end{abstract}

Discussion: This study will address a critical but as yet unanswered research question: to identify an effective way to reduce psychological distress; and improve the quality of life for men with advanced prostate cancer.

Trial registration: ACTRN12612000306819

Keywords: Prostate cancer, Randomised controlled trial (RCT), Supportive care, Mental health, Psychological distress, Quality of life, Health outcomes

\section{Background}

Prostate cancer is the most common male cancer in developed countries (excluding non-melanoma skin cancer). In 2007, 19,403 Australian men were diagnosed with prostate cancer [1]. Approximately $10-15 \%$ of men with prostate cancer have locally advanced or metastatic prostate cancer at diagnosis [2] and a further $20-40 \%$ of men

\footnotetext{
* Correspondence: suzanne.chambers@griffith.edu.au

${ }^{1}$ Griffith Health Institute, Griffith University, Gold Coast, QLD 4222, Australia

${ }^{2}$ Cancer Council Queensland, Brisbane, Australia

Full list of author information is available at the end of the article
}

with localised cancer at diagnosis experience recurrence or progression after treatment [3]. Men with advanced prostate cancer face additional physical and psychological challenges compared to men with localised disease. The iatrogenic effects of hormonal ablation, the main treatment for advanced disease, include mood disturbance, cognitive impairment, fatigue, and sexual dysfunction [4]. By comparison to men with localised prostate cancer, men with advanced disease report higher levels of psychological distress and poorer quality of life (QoL) [5,6]; and have an increased risk of suicide $[7,8]$. Hence research into

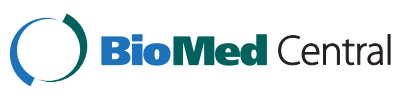


psychological intervention to maximise psychological adjustment is crucial for these men.

To date, no psychological intervention research specifically targeting men with advanced prostate cancer has been reported [9]. In this study we propose using the cognitive behavioural approach of MindfulnessBased Cognitive Therapy (MBCT) as relevant to this patient group. Mindfulness involves open awareness of current experience and the intention to observe habits of reacting to difficulties as they arise. Over time, this practice leads the person to gain the ability to be less reactive to difficult experiences and approach equanimity regarding the content of the illness experience, including negative emotions and thoughts. MBCT specifically targets the cognitive processes associated with depression by encouraging participants to disengage from reactive and ruminative states of mind, such as those that are commonly reported by cancer patients [10,11]. Qualitative studies of cancer patients who have taken part in mindfulness courses have identified positive changes in acceptance, self-control, personal growth, shared experience and self-regulation as outcomes of mindfulness practice [12,13]. In a pilot study of group MBCT conducted with 19 men with advanced prostate cancer, men reported significant changes in both general psychological and cancerspecific distress [14]. Qualitative data revealed that acceptance of and learning from other group members were key aspects of the group context that contributed to acceptance of progressive disease. Thus, in the context of MBCT the group setting appears important as a contributor to acceptance of cancer through peer learning and modelling.

Accordingly, in this trial we apply a tele-based MBCT group intervention to decrease anxiety and depression and cancer-specific distress in men with advanced prostate cancer.

\section{Methods/design}

The study has two arms: 1) MBCT delivered by teleconference over eight weekly sessions and (2) patient education.

It is hypothesised that 3, 6 and 9 months after recruitment and commencement of the intervention:

1. Relative to men who receive patient education, men who receive MBCT will have lower anxiety and depression.

2. Relative to men who receive patient education, men who receive $\mathrm{MBCT}$ will have lower cancer-specific distress.

3. Relative to men who receive patient education, men who receive $\mathrm{MBCT}$ will have higher mindfulness.

4. Intervention-driven improvements in psychological outcomes will be mediated by mindfulness skills.

\section{Group condition}

Patient Education

Patient education consists of the man's standard medical management and a package containing existing evidencebased patient education materials.

\section{Mindfulness-Based Cognitive Therapy}

The Mindfulness-Based Cognitive Therapy group intervention $(\mathrm{MBCT})$ follows a cancer-specific manual based on Segal, Williams and Teasdale's [15] manual for MBCT, with novel specific components developed for men with advanced prostate cancer in our pilot study [14]. The sessions are facilitated by health professionals with experience in oncology and professional training in МВCT. The program has been further developed to be suitable for telephone delivery. For example, facilitators have been trained to use explicit communication such as encouraging group members to say their name before contributing, listen closely to the tone of participants' voices, give extra time for responses, and have explicit rules for how the group will interact. Each session runs for 1.25-h and only short (up to $15 \mathrm{~min}$ ) meditations are provided during the group phone sessions to support group engagement and alleviate practical concerns such as holding the telephone receiver. As well, the material in participant workbooks has been elaborated to provide session plans, so participants can navigate phone sessions more easily, and interactive worksheets are provided to help keep group discussions on task. The program includes eight weekly group teleconferences, and each participant has an individual introductory call with their facilitator to allow them to connect with the facilitator, prepare them for the program and to enhance motivation. Participants are provided with a handbook summarising each weekly session; self-help materials including Jon Kabat Zinn's Full Catastrophe Living [16]; and an audio recorded meditation on compact disc. Daily home practice of mindfulness meditation is strongly encouraged; with participants asked to engage in one of the four $35 \mathrm{~min}$ practices depending on the stage of the course (the body scan, moving meditation, mixed mindfulness meditation, silent practice with bells at $5 \mathrm{~min}$ intervals). Finally, as in our pilot study, peer interaction is directed towards support for the learning of mindfulness skills and mutual support in facing the challenges of advanced prostate cancer.

\section{Participants}

Recruitment is being undertaken through clinicians in the Australian and New Zealand Urogenital and Prostate Cancer Trials Group and in major treatment centres in Queensland, New South Wales, Victoria and Western Australia. Other recruitment avenues include the distribution of information through prostate cancer support 
groups and media promotion. The research team who contact potential participants after referral to the study obtains informed written consent. Figure 1 illustrates the recruitment, intervention and data collection process.

Inclusion criteria are that the men must: (1) have proven metastatic disease or castration resistant biochemical progression; (2) be able to read and speak English; (3) have no previous history of head injury, dementia or psychiatric illness; (4) have no other concurrent cancer; (5) have phone access.

Approximately 190 men will be recruited to the study (allowing 30\% attrition from treatment; 65 men in each condition will complete final assessments). This sample size would comfortably exceed $80 \%$ power to detect a moderate to large effect over four assessment points.

\section{Study integrity}

Ethical approval has been obtained from the Griffith University Human Research Ethics Committee (Approval:
PSY/15/12/HREC) and Metro-North - The Prince Charles Hospital Human Research Ethics Committee (HREC/12/QPCH/101). The study design is guided by the CONSORT criteria [17]. Randomisation to study condition occurs following the completion of baseline assessment. Assessments are by self-report pen and paper measures and project staff tracking assessments are blinded to condition where possible. Randomisation occurs in blocks of 14, with each condition randomly generated 7 times within each block to ensure an unpredictable allocation sequence with equal numbers of men in each condition at the completion of each block; and sufficient men to form a tele-based group (of 7) in the MBCT condition. Randomisation occurs within Queensland-Western Australia and New South Wales-Victoria dyads to coincide with a twostage commencement of recruitment. The randomisation sequence is undertaken by the project manager and concealed from investigators. The group sessions are audiotaped with $15 \%$ reviewed to ensure adherence to an

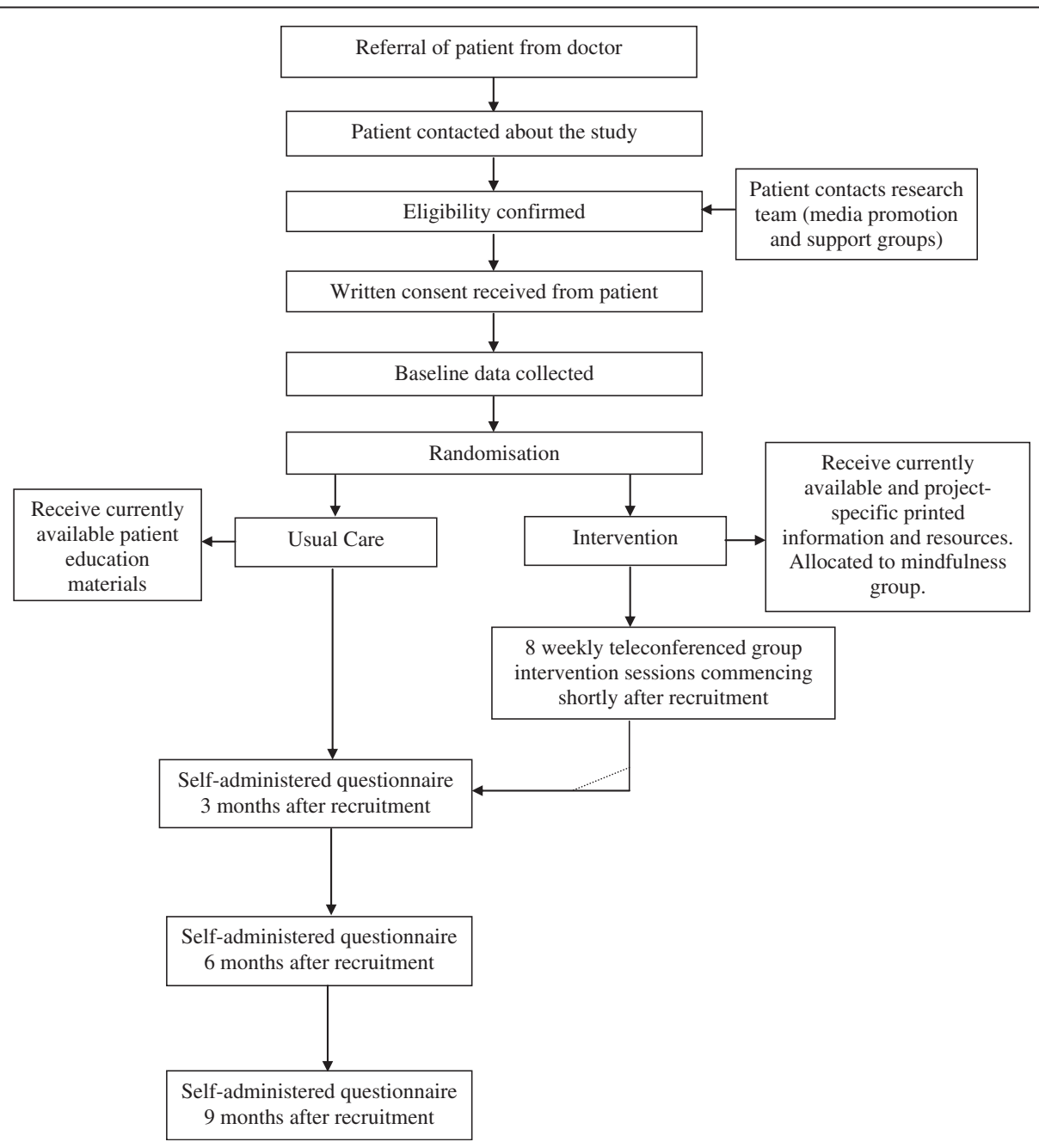

Figure 1 Flowchart of recruitment, intervention and assessment. 
MBCT approach. Analyses will be conducted on the basis of intention to treat.

\section{Measures}

A series of previously validated and reliable self-report measures are administered by mail to men at four time points: baseline/recruitment and at 3, 6, and 9 months after recruitment and intervention commencement. Mindfulness skills are included as potential mediators of intervention effect. Primary outcomes are anxiety, depression and cancer-specific distress. Secondary outcomes are global QoL and benefit finding. Disease variables (e.g. cancer grade, stage, time since diagnosis, time since treatment) will be assessed through medical records review.

\section{Mediators}

Mindfulness: The Five Facet Mindfulness Questionnaire (FFMQ) [18] is being used to measure the participants' engagement with the principles of mindfulness.

Adherence to Practice: Adherence to mindfulness practice is assessed by participants completing a daily home practice form.

\section{Primary outcome variables}

Anxiety and Depression: The Brief Symptom Inventory-18 [19] is providing a global measure of current psychological distress with subscale scores for anxiety, depression, and somatisation.

Cancer-Specific Distress: The Impact of Events Scale (IES) $[20,21]$ and the PSA Anxiety subscale of the Memorial Anxiety Scale for Prostate Cancer (MAX-PC) [22] is being used to measure men's cancer-specific distress.

\section{Secondary outcome variables}

Quality of Life: The Functional Assessment of Cancer Therapy - Prostate (FACT-P) [23] is being used to assess the men's perceived global quality of life across 5 domains: physical, social/family, emotional, functional well-being, and prostate cancer-specific concerns.

Benefit Finding: Positive adjustment is being measured with the Posttraumatic Growth Inventory assessing perceived positive outcomes resulting from a diagnosis of cancer [24].

\section{Statistical analyses}

The study is a two-condition randomised controlled trial with repeated measures across time and continuous outcome variables. The analysis of longitudinal differences in outcome will be by multilevel (mixed) modelling (MLM). These procedures allow the testing of typical group level predictions such as Hypotheses 1 to 3 that men in the intervention condition will have better outcomes than the patient education group. However, by incorporating the hierarchical structure of assessment points nested within individual men they further permit the true assessment of individual change in psychological outcomes and of potential mediators of such change (Hypothesis 4). Consequently (and unlike traditional approaches), this model deals with the heterogeneity of responses, such as that expected in the outcomes of the proposed study, by allowing such variation as random effects within the model. MLM has the advantage of allowing use of all available data points, which maximizes power to detect effects and reduces bias owing to missing data in longitudinal studies.

\section{Discussion}

The study will provide recommendations about effective psychological interventions to reduce anxiety, depression and cancer-specific distress in men with advanced prostate cancer. To our knowledge, to date no psychological intervention studies have targeted men with advanced prostate cancer specifically; or trialled remotely delivered mindfulness interventions in cancer populations [9]. This research will overcome these limitations. If proven effective, the intervention will be able to be utilised in a range of settings including broad reach tele-health support programs; and through support services and support groups internationally. This means that project outputs will be immediately translatable into practice to reduce psychological distress and improve the quality of life of men with advanced prostate cancer.

\section{Competing interests}

The authors declare that they have no competing interests.

\section{Authors' contributions}

SKC, DS and MB developed the study concept and aims and initiated the project. SL, EF, SC, RM, SO, MF and RAG assisted in further development of the protocol. SKC was responsible for drafting the manuscript. SKC, DS, EF, SC, RM and SO will implement the protocol and oversee collection of the data. All authors contributed to the final manuscript.

\section{Acknowledgements}

This project was funded by the Australian National Health and Medical Research Council (ID APP1024989), and undertaken in partnership with the Australian and New Zealand Urogenital and Prostate (ANZUP) Cancer Trials Group and Cancer Council Queensland and New South Wales. We gratefully acknowledge the support of the Prostate Cancer Foundation of Australia; the Urological Society of Australia and New Zealand; of Professor lan Davis, Dr Peter Heathcote, Professor Nigel Spry, Associate Professor Martin Stockler, and Dr John Yaxley as associate investigators; and of Mr Bill McHugh and $\mathrm{Mr}$ Peter Dornan as consumer advisors.

\section{Author details}

${ }^{1}$ Griffith Health Institute, Griffith University, Gold Coast, QLD 4222, Australia. ${ }^{2}$ Cancer Council Queensland, Brisbane, Australia. ${ }^{3}$ Prostate Cancer Foundation of Australia, Sydney, Australia. ${ }^{4}$ Australian and New Zealand Urogenital and Prostate Cancer Trials Group, Sydney, Australia. ${ }^{5}$ Cancer Council NSW, Sydney, Australia. ${ }^{6}$ University of New South Wales, Sydney, Australia. ${ }^{7}$ Department of Public Health, Temple University, Philadelphia, USA. ${ }^{8}$ Mind Potential, Sydney, Australia. ${ }^{9}$ School of Psychology, Griffith University, Brisbane, Australia.

${ }^{10}$ Department of Surgery, Monash University, Melbourne, Australia. ${ }^{11}$ Royal Melbourne Hospital, Melbourne, Australia. ${ }^{12}$ University of Queensland Centre for Clinical Research, University of Queensland, Brisbane, Australia. 
${ }^{13}$ Department of Urology, Royal Brisbane and Women's Hospital, Brisbane, Australia.

Received: 14 January 2013 Accepted: 21 February 2013 Published: 26 February 2013

\section{References}

1. Australian Institute of Health and Welfare, Australasian Association of Cancer Registries: Cancer in Australia: An overview, 2006, Cancer Series AlHW cat no CAN 32. Canberra: Australian Institute of Health and Welfare; 2007.

2. Alam N, You H, Banks C, Baker D, Bishop J: Prostate Cancer in New South Wales. Sydney: Cancer Institute NSW; 2009.

3. Han M, Partin AW, Zahurak M, Piantadosi S, Epstein JI, Walsh PC Biochemical (prostate specific antigen) recurrence probability following radical prostatectomy for clinically localized prostate cancer. J Urol 2003, 169(2):517-523.

4. Australian Cancer Network Management of Metastatic Prostate Cancer Working Party: Clinical practice guidelines for the management of locally advanced and metastatic prostate cancer. Sydney: Cancer Council Australia and Australian Cancer Network; 2010.

5. Eton DT, Lepore SJ: Prostate cancer and health-related quality of life: a review of the literature. Psychooncology 2002, 11(4):307-326.

6. Bloch S, Love A, Macvean M, Duchesne G, Couper J, Kissane D: Psychological adjustment of men with prostate cancer: a review of the literature. Biopsychosoc Med 2007, 1:2.

7. Bill-Axelson A, Garmo H, Lambe M, Bratt O, Adolfsson J, Nyberg U, Steineck $G$, Stattin P: Suicide risk in men with prostate-specific antigen-detected early prostate cancer: a nationwide population-based cohort study from PCBaSe Sweden. Eur Urol 2010, 57(3):390-395.

8. Fang F, Keating NL, Mucci LA, Adami H, Stampfer MJ, Valdimarsdottir U, Fall $\mathrm{K}$ : Immediate risk of suicide and cardiovascular death after a prostate cancer diagnosis: cohort study in the United States. J Natl Cancer Inst 2010, 102(5):307.

9. Chambers SK, Pinnock C, Lepore SJ, Hughes S, O'Connell DL: A systematic review of psychosocial interventions for men with prostate cancer and their partners. Patient Educ Couns 2011, 85(2):e75-88.

10. Lau MA, Segal ZV, Williams JMG: Teasdale's differential activation hypothesis: implications for mechanisms of depressive relapse and suicidal behaviour. Behav Res Ther 2004, 42:1001-1017.

11. Sheppard LC, Teasdale JD: How does dysfunctional thinking decrease during recovery from major depression? J Abnorm Psychol 2004, 113:64-71.

12. Dobkin PL: Mindfulness-based stress reduction: what processes are at work? Complement Ther Clin Pract 2008, 14(1):8-16.

13. McKenzie MJ, Carlson LE, Munoz M, Speca M: A qualitative study of self perceived effects of mindfulness based stress reduction in a psychosocial oncology setting. Stress and Health 2007, 23(1):59-69.

14. Chambers SK, Foley E, Galt E, Ferguson M, Clutton S: Mindfulness groups for men with advanced prostate cancer: a pilot study to assess feasibility and effectiveness and the role of peer support. Support Care Cancer 2012, 20(6):1183-1192.

15. Segal ZV, Williams JMG, Teasdale JD: Mindfulness-based cognitive therapy for depression: a new approach to preventing relapse. New York: Guilford Press; 2002.

16. Kabat-Zinn J: Full catastrophe living: using the wisdom of your body and mind to face stress, pain, and illness. New York: Delacorte; 1990.

17. Schulz KF, Altman DG, Moher D: CONSORT 2010 Statement: updated guidelines for reporting parallel group randomised trials. BMC Med 2010, 8:18.

18. Baer RA, Smith GT, Hopkins J, Krietemeyer J, Toney L: Using self-report assessment methods to explore facets of mindfulness. Assessment 2006, 13(1):27-45

19. Derogatis LR: BSI-18: Administration, Scoring and Procedures Manual. Minneappolis MN: National Computer Systems; 2000.

20. Horowitz M, Wilner N, Alvarez W: Impact of event scale: a measure of subjective stress. Psychosom Med 1979, 41(3):209-218.

21. Weiss DS, Marmar CR: The Impact of Events Scale - Revised. In Assessing Psychological Trauma and PTSD. Edited by Wilson JP, Keane TM. New York: Guilford Press; 1997:399-411.

22. Roth AJ, Rosenfeld B, Kornblith AB, Gibson C, Scher HI, Curley-Smart T, Holland JC, Breitbart W: The memorial anxiety scale for prostate cancer: validation of a new scale to measure anxiety in men with prostate cancer. Cancer 2003, 97(11):2910-2918.
23. Cella DF, Tulsky DS, Gray G, Sarafian B, Linn E, Bonomi A, Silberman M, Yellen SB, Winicour P, Brannon J, et al: The functional assessment of cancer therapy scale: development and validation of the general measure. J Clin Oncol 1993, 11(3):570-579.

24. Tedeschi RG, Calhoun LG: The posttraumatic growth inventory: measuring the positive legacy of trauma. J Trauma Stress 1996, 9(3):455-471.

doi:10.1186/1471-2407-13-89

Cite this article as: Chambers et al: A randomised controlled trial of a mindfulness intervention for men with advanced prostate cancer. $B M C$ Cancer 2013 13:89.

\section{Submit your next manuscript to BioMed Central and take full advantage of:}

- Convenient online submission

- Thorough peer review

- No space constraints or color figure charges

- Immediate publication on acceptance

- Inclusion in PubMed, CAS, Scopus and Google Scholar

- Research which is freely available for redistribution

Submit your manuscript at www.biomedcentral.com/submit
C Biomed Central 